\title{
Management of COPD in Primary Care in Leicestershire
}

\author{
Aye Ma Lwin, Robert K. McKinley*
}

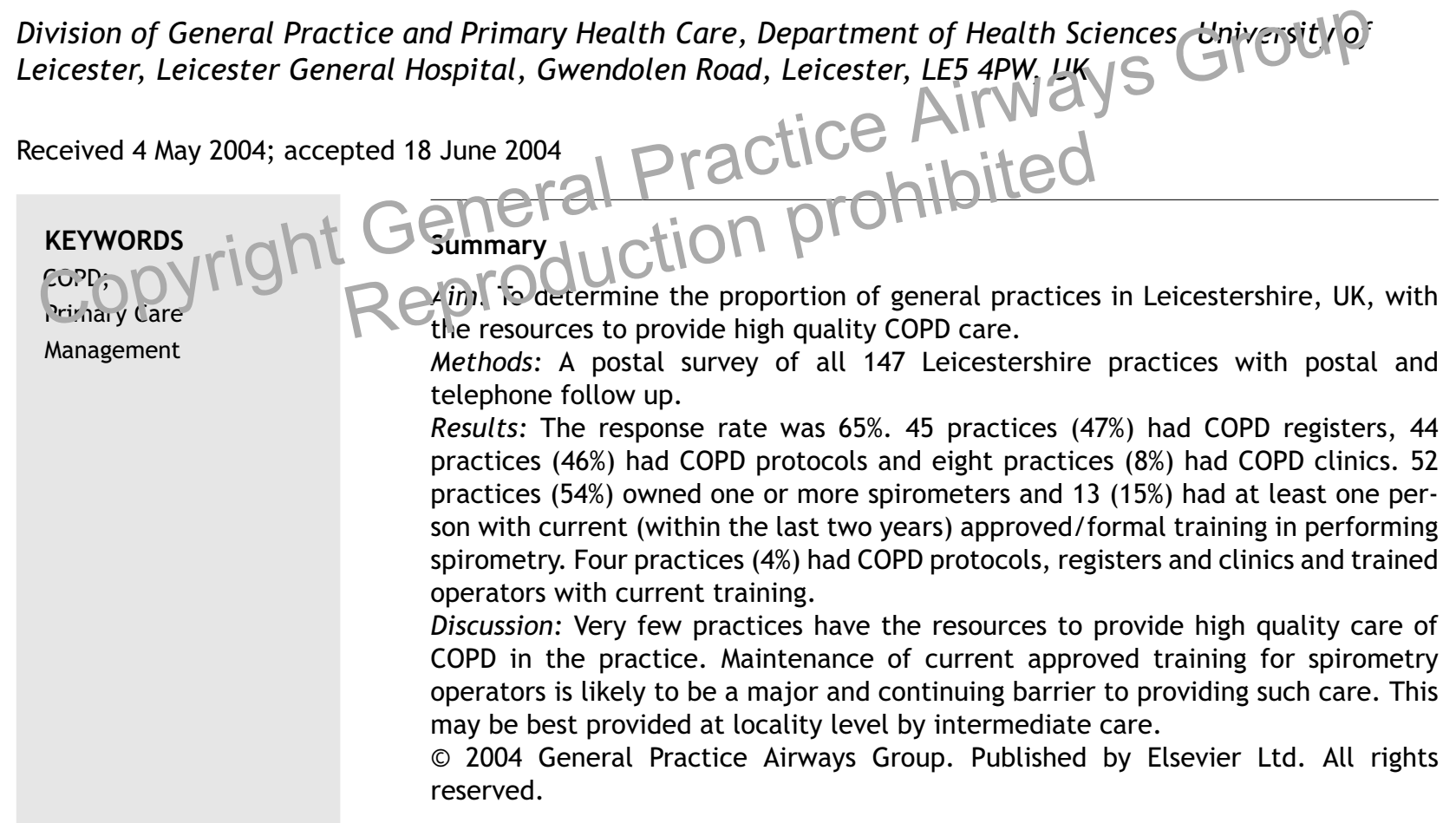

\section{Introduction}

Chronic obstructive pulmonary disease (COPD) is a common treatable condition, is currently the fourth leading cause of death in the world [1] and affects $11 \%$ of people aged 45 and over in the United Kingdom [2]. It is estimated that two or three times this number remain undiagnosed $[2,3]$. Due to similarities in clinical presentation COPD

* Corresponding author. Tel.: +44 116258 4367; fax: +441162584982.

E-mail address: rkm@le.ac.uk (R.K. McKinley). is often confused with asthma [4], but important differences exist with respect to aetiology, pathology, management and prognosis. Reduction of morbidity and mortality in people with COPD requires an accurate diagnosis and optimal management informed by clinical guidelines based on the latest available evidence [2].

Such care could be provided in general practices [5]. $85 \%$ of people with chronic respiratory disease receive their care from their general practitioner [6]. General practices are in an ideal position to develop and maintain accurate COPD disease registers. These are necessary to facilitate regular 
call and recall for planned management, which is based on clinical assessment and spirometry which requires relatively 'low tech' and inexpensive equipment. Finally, general practices are usually more accessible to patients than secondary care centres. Nevertheless, this all requires practices to have accurate disease registers, dedicated clinical time, evidence-based protocols and access to quality-assured spirometry. Such quality- assured spirometry requires an appropriately calibrated spirometer with a volume/time display and an operator who has had approved training during the previous two years [7].

We report the results of a survey which aimed to assess whether practices in one English county have the facilities to provide high quality care of COPD.

\section{Methods}

A cross-sectional quantitative postal questionnaire survey of all 147 general practices in Leigester shire was carried out between Ja liov vand ivarch 2002 A questionnaire ias developed to adtitess the studv aims by semi-structired ineriews with general practitioners and practice nurses and by adaptation of a questionnaire used in Devon and Cornwall $[8,9]$. The questionnaire was piloted on five doctors and six nurses in volunteer practices before it was administered. To improve response rates, the lead respiratory nurse and doctor in each of the 147 practices were identified by telephone contact prior to questionnaire administration and the questionnaire was returned to the University Department of General Practice \& Primary Health Care. A reminder letter with a second questionnaire was sent after six weeks of non-response. The questionnaire was then shortened and administered via telephone to non-responding practices after 12 weeks.

\section{Results}

A response was received from 96 of 147 practices $(65 \%)$, of which 78 responded by questionnaire and 18 by telephone to the shortened questionnaire. Of the informants, 31 were practice managers, 61 practice nurses and 4 general practitioners. Of the practices from which we received a response, $47(49 \%)$ were city, $28(29 \%)$ market town and 21 (22\%) village or rural practices; the corresponding figures for non-responding practices were $24(47 \%)$, $16(31 \%)$ and 11 (22\%), (Chi Square 0.1, 2 degrees of freedom, $p=1.0$ ). The median (interquartile range) numbers of partners in responding and non-responding practices were four $(1-5)$ and two $(1-4)$ respectively (Mann Whitney- $U=2166$, $p=0.2)$. Of the 96 responding practices, $45(47 \%)$ reported that they had COPD registers of which 37 were compiled using searches of computer and paper medical records for a record of a diagnosis of COPD or relevant prescription. Forty-four of 96 practices (46\%) had COPD protocols, and 27 had derived them from the BTS guidelines, although 66 of the responding practices did not respond to this item. Eight practices had dedicated COPD clinics and a further 36 had combined COPD/Asthma clinics. Conversely, 85 responding practices had asthma registers and 36 had dedicated asthma clinics.

Fifty-two responding practices offered in-house spirometry while 33 had access to hospital spirometry services either directly (six practices) or by referral (27 practices). Details of thel rake and model of spirometers wee available for 36 practices Dat: icr the 14 remaining practices who tated that they had a splrometer were gathered by telephonc vien the questions on the make and mind (-1) cf spirometers were omitted. Spirometers in eight of these 36 practices had volume/time displays. Twenty of these 36 practices either did not know whether their spirometers required calibration or had never calibrated them.

Of the 89 people who performed spirometry in these practices, only $37(41 \%)$ had had formal training; $13(15 \%)$ in 11 practices had had formal training within the past two years, and 35 nurses in 20 practices had undertaken training in the last five years.

Four practices $(4 \%)$ had COPD registers, protocols, clinics and trained operators with current training. This number was unchanged when the practices which had direct external access to spirometry were included or if the definition of current training was extended to include that undertaken in the last five years.

Spirometry was most often used for establishing or excluding a diagnosis of COPD in people with respiratory symptoms (45 of 56 responding practices, $88 \%)$. Only 17 of 56 practices (30\%) used it for screening for COPD. $70 \%$ and $60 \%$ of responding practices used spirometry for reversibility testing to bronchodilators and corticosteroids, respectively.

\section{Discussion}

Only one in six participating practices had at least one person with current training (i.e. training within the last two years) in performing spirometry and one in 25 may have had the facilities required to provide high quality COPD care-that is, a 
disease register, a protocol, clinics and access to quality-assured spirometry. When we expand our definition of practices which are equipped to provide high quality COPD care to those with external access to spirometry, still only four per cent fulfil our definition.

We have adopted the stance that high quality COPD care requires protocols to inform and guide clinical care, COPD registers to facilitate call and recall so that all those on the register can be offered therapy and advice on slowing of disease progression, clinics to ensure that the time for such activity is available to both patients and their clinical carers, and quality-assured spirometry. We acknowledge that this is stringent and that it is possible for well-trained motivated clinical staff to provide high quality care for individual patients without these facilities; nevertheless, we believe that it is not possible to ensure adequate population coverage without these facilities and we doubt that it would be possible for the "typical practice" to provide the ftuctured multi-disciplinary care re om i1enceú by NICE [2] Notwithstanding (h) stringency of our prerlecilisites fo higb qua ity COPD cire, u: Clss acknowledge that we have not verified the quality of the practices' protocols or disease registers. Indeed, we suspect that the disease registers are inaccurate because they were mainly constructed through review of practice records without spirometric validation of the diagnosis. Similarly, the existence of a COPD clinic does not ensure that good care is provided but we would argue that it is more likely in practices which have committed this resource. Finally, when judging the quality and currency of spirometry training we have adopted the stance of the Association of Respiratory Technology and Physiology, namely that a certificate of proficiency in performing spirometry is valid for two years [7]. Although this may be a high standard, we know that spirometry performed by untrained operators is unreliable [10], although training does not necessarily guarantee quality [11].

The two previous surveys of spirometry in primary care identified that the majority of primary care staff performing spirometry were untrained, but did not establish the currency of the training for those who had actually received training $[8,12,13]$. A similar proportion of practices in the current and previous studies [8] were using practice-based spirometry for the diagnosis of COPD. It is of some concern that important clinical decisions are being based on results of uncertain validity.

The major weakness of this study is our modest response rate. In spite of written and telephone follow up, 35\% of practices did not respond.
Nevertheless, it is likely that those practices which did respond are the more "respiratory aware" practices and that the prevalence of protocols, registers, clinics and trained operators is likely to be lower amongst non-responders. Furthermore, it is likely that practices have overstated rather than underestimated their capacity. Our estimate that $4 \%$ of the population of practices are equipped to provide high quality COPD care is more likely to be an over-estimate then an underestimate. Although this study was conducted in a single English county we believe that the results are representative of United Kingdom general practice, a stance supported by the similarities between our data and the previous studies (above) $[8,12,13]$.

The Quality and Outcomes markers in the new United Kingdom General Practitioner contract include the care of COPD [1] 7 The pussession of disease register and the use of spirometry in the diagnos: and nonitoring of COPD are quality indicators. These data ic ive important implications for the valicily of these quality markers.

We be'ieve that these data support the move to develop intermediate care services for people with COPD $[15,16]$. It makes the provision of clinical time and expertise supported by high quality evidence-based protocols and quality-assured spirometry much more straightforward by minimising the number of staff whose training requires updating. Practices will still have responsibility for identifying people at risk of COPD by, for example, identifying those aged 40 who smoke; facilitating the invitation of those patients to intermediate services for initial screening, definitive diagnosis and continuing review; and by maintaining their COPD registers. It will, however, free individual practices from the need to develop, and importantly maintain, the expertise to provide such care.

\section{Acknowledgements}

We would like to thank the general practitioners and practice nurses and managers who responded to our study and Dr Rupert Jones for providing us with copies of his questionnaires. We would also like to thank the GPIAG for its financial support of this project.

\section{References}

[1] World Health Organisation. Deaths by cause, sex and mortality stratum in WHO regions: estimates for 1999. The World Health Report, Annex Table 3. 2000.

[2] National Coordinating Centre for Chronic Conditions. Chronic obstructive pulmonary disease: management of 
adults with chronic obstructive pulmonary disease in primary and secondary care - Draft. http://www.nice.org. uk/pdf/COPD_full_guideline_first_consultation.pdf. 28-82003.

[3] Siafakas NM, Vermeire P, Pride NB, Paoletti P, et al. Optimal assessment and management of chronic obstructive pulmonary disease (COPD). The European Respiratory Society Task Force. Eur Respir J 1995;8:1398-420.

[4] Griffiths C, Feder G, Wedzicha J, Foster G, et al. Feasibility of spirometry and reversibility testing for the identification of patients with chronic obstructive pulmonary disease on asthma registers in general practice. Respir Med 1999;93:903-8.

[5] Van Schayck OCP, Rabe KF, Rudolf M. COPD: The role of primary care in effective diagnosis, treatment and management. Primary Care Respiratory Journal 2003;12:1620.

[6] Morbidity Statistics from General Practice. Fourth National Survey 1991-1992. Royal College of General Practitioners, Office of Population Censuses and Surveys, and the Department of Health, London: 1995.

[7] Cooper B, Spiro S, Britton M. Joint statement from the Association for Respiratory Technology and Physiology, the British Thoracic Society and the British Lung Foundation for World COPD Day. http://fp.artpweb.f9.co. IK/ archive/2003/News/ARTP-BTS-BLF\%20World\%2FCOPl)\% 20Spiro\%20Statement.pdf. 19-r1-200.
[8] Jones RCM, Freegard S, Reeves M, et al. The role of the practice nurse in the management of chronic obstructive pulmonary disease (COPD). Primary Care Respiratory Journal 2001;10:106-8.

[9] Jones RCM, Freegard S, Reeves $M$, et al. The role of the practice nurse in the management of asthma. Primary Care Respiratory Journal 2001;10:109-11.

[10] Eaton T, Withy S, Garrett JE, et al. Spirometry in primary care practice: The importance of quality assurance and the impact of spirometry workshops. Chest 1999;116:416-23.

[11] den Otter JJ, Knitel M, Akkermans RPM, et al. Spirometry in general practice: The performance of practice assistants scored by lung function technicians. Br J Gen Pract 1997;47:41-2.

[12] Dowson LJ, Yeung A, Allen MB. General practice spirometry in North Staffordshire. Monaldi Arch Chest Dis 1999;54:186-8.

[13] Dowson LJ. Most practices would use open access spirometry in hospitals. [Letter]. BMJ 1998;317:209.

[14] The NHS confederation, GMSC. Investing in general practice. New GMSC contract. London: The NHS con'e teration and the BMA, 2003.

[15] Dhillon R. SOs vith sefcicl interests. Primary Care Respiria iny Joural 2002;11:89.

16] ( $r$ uify du-Jones $K$. The frar it work for general practitioners with a snedial in ei $\in$ st in resplratory medicine. Primary Care Res, irato y Juurnal 2003;12:35.

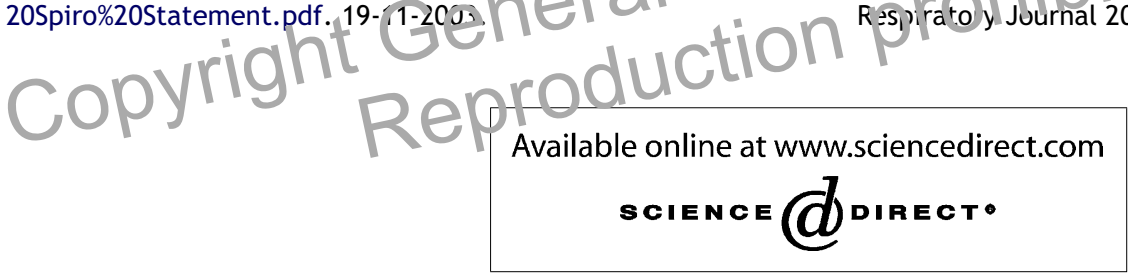

Available online at http://www.thepcrj.com 